\title{
Does ball carrier technique influence tackler head injury assessment risk in elite rugby union?
}

\section{Gregory J Tierney, Karl Denvir, Garreth Farrell \& Ciaran K Simms}

To cite this article: Gregory J Tierney, Karl Denvir, Garreth Farrell \& Ciaran K Simms (2019) Does ball carrier technique influence tackler head injury assessment risk in elite rugby union?, Journal of Sports Sciences, 37:3, 262-267, DOI: 10.1080/02640414.2018.1494952

To link to this article: https://doi.org/10.1080/02640414.2018.1494952

\section{曲 Published online: 03 Jul 2018.}

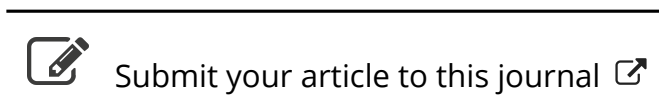

Цll Article views: 218

View Crossmark data ¿

Citing articles: 2 View citing articles $\longleftarrow$ 


\title{
Does ball carrier technique influence tackler head injury assessment risk in elite rugby union?
}

\author{
Gregory J Tierney (1) ${ }^{a}$, Karl Denvir ${ }^{b}$, Garreth Farrell ${ }^{b}$ and Ciaran K Simms ${ }^{a}$ \\ aTrinity Centre for Bioengineering, Trinity College Dublin, Dublin, Ireland; bLeinster Rugby, Dublin, Ireland
}

\begin{abstract}
The aim of this study was to use video evidence of tackles in elite level rugby union to identify ball carrier proficiency characteristics, for both lower and upper body tackles, that have a higher propensity to result in Head Injury Assessments(HIA) for the tackler. HIA $(n=74)$ and non-HIA tackles $(n=233)$ were categorised as either front-on or side-on upper or lower body tackles and scored for ball carrying proficiency characteristics. Side-on tackles included tackles from behind. A Chi-Square test $(p<0.05)$ and Cramer's V were calculated to compare proficiency characteristics in HIA and non-HIA cases. For front-on upper body tackles, the ball carrier "fending into contact" ( $p<0.01$;ES = Moderate) and "explosiveness on contact" ( $p=0.04$;ES = Moderate) had a higher propensity to result in a HIA for the tackler. Fending into contact was exhibited in $47 \%$ of all upper body Tackle front-on HIA cases. The fending arm contacted the tackler's head in $67 \%$ of these cases. Fending into contact can potentially be dangerous and therefore emphasis should be placed on safe fending during tackle-based training drills. Referees should also be alert to arm-to-head contact during the fend. Given the low number of ball carrier characteristics identified, focus should be placed on tackler characteristics for HIA prevention strategies.
\end{abstract}

ARTICLE HISTORY

Accepted 26 June 2018

KEYWORDS

Concussion; Head Impact; Tackling; Injury Prevention

\section{Introduction}

Tackling is the main cause of contact in rugby union (Fuller, Brooks, Cancea, Hall, \& Kemp, 2007) with some players making over 30 tackles per game (Deutsch, Kearney, \& Rehrer, 2007). Unfortunately, it is also the most common cause of head injuries (Fuller, Laborde, Leather, \& Molloy, 2008; Fuller, Taylor, \& Raftery, 2015; Quarrie \& Hopkins, 2008; Tierney, Lawler, Denvir, McQuilkin, \& Simms, 2016). For the sixth consecutive season, concussion was the most commonly reported match injury for English Premiership rugby union (incidence rate of 20.9/1000 player hours, contributing to $22 \%$ of all match injuries during the 2016-17 season) (Union, 2018). In one season alone, one study found that $23 \%$ of elite level Rugby Union participants suffered a concussion (Shuttleworth-Edwards et al., 2008). It is well understood that both correct tackler and ball carrier technique are necessary for successful and safe participation in rugby union (Hendricks \& Lambert, 2010, 2014; Tierney, Denvir, Farrell, \& Simms, 2018a, 2018c; Tierney, Krosshaug, Wilson, \& Simms, 2015), and that incorrect tackle technique is a risk factor for injury (Burger et al., 2016; Hendricks et al., 2015).

Video analysis studies have provided valuable information on injury (Burger et al., 2016; Quarrie \& Hopkins, 2008) and concussion (Fuller et al., 2015; Hendricks et al., 2015, 2016) risk factors in rugby union. This has also been the case for concussion injury research in other sports such as rugby league (Gardner et al., 2015), ice hockey (Hutchison, Comper, Meeuwisse, \& Echemendia, 2013) and soccer (Andersen, Arnason, Engebretsen, \& Bahr, 2004). A recent study (Tierney et al., 2016) on direct head impacts in elite level rugby union reported that the tackle phase of play accounted for $60 \%$ of direct head impacts and that the tackler was the head impacted player in $97 \%$ of these cases.

It appears that the tackler is at most risk of a direct head impact, and hence concussion, in the tackle. As a result, Tierney et al. (Tierney, Denvir, Farrell, \& Simms, 2018b) performed a video analysis study of elite level match play to identify tackler proficiency characteristics that had a lower propensity to result in a Head Injury Assessment (HIA) for the tackler. The HIA was first introduced in 2012 by World Rugby as the pitch side assessment process for concussion injuries (Fuller, Kemp, \& Decq, 2015) and has previously been described in detail (Fuller, Kemp, \& Raftery, 2017). In brief, the aim of the HIA is to create a standardised tool for the medical assessment of suspected concussion injuries in rugby union (McCrory et al., 2005). During a game, a player enters the HIA protocol by displaying on-field signs and symptoms of concussion (McCrory et al., 2005). The HIA assesses a range of concussive symptoms including memory difficulties, cognitive ability, balance and player discomfort. In the HIA, if a player's score is positive, they are removed from play and must follow the return-to-play protocol (Fuller et al., 2017). Of the 24 diagnosed concussions at the 2015 Rugby World Cup, 19 were first suspected on the field using the HIA protocol (Fuller et al., 2017). The remaining 5 were suspected post-match. A total of 39 onfield HIAs were conducted during the 2015 Rugby World Cup (Fuller, Fuller, Kemp, \& Raftery, 2017). It is clear that a reduction in tackle related HIAs would have a strong influence on concussion injury reduction. Tierney et al. (Tierney et al., 2018b) 
found several tackler proficiency variables that had a lower propensity to result in a HIA for the tackler, especially "identify/track ball carrier onto shoulder", "head up and forward/face up", "straight back, centre of gravity forward of support base" and "head placement on correct side of ball carrier". The results from the study provided an evidence-base at the elite level to assist coaches in developing and implementing HIA prevention strategies for the tackler.

However, this study did not assess ball carrier proficiency characteristics and how these might affect tackler HIA risk. Rapidly changing and dynamic tackle scenarios require tacklers to make split-second decisions when reacting to ball carriers who frequently adjust their speed and direction to avoid or break contact. Part of the skill of ball carrying is to deceive the opposition tackler and thereby ensure an effective tackle cannot be executed, however, it may also jeopardise tackler safety. It is possible that specific ball carrying characteristics are linked to HIA causation for the tackler for both upper body and lower body tackles, the details of which are unknown.

Accordingly, the aim of this study is to use match video evidence of tackles in elite level rugby union to identify ball carrier characteristics that result in HIA for the tackler. This study has the potential to further clarify tackle-related head injury mechanisms. This in turn can allow effective HIA prevention strategies to be developed.

\section{Methods}

\subsection{Research design and data collection}

A qualitative observational case-control study design was used on a cohort of professional men rugby union players to identify specific ball carrier characteristics (Table 1-4) associated with HIA and non-HIA tackles. A tackle was defined as "when the ball-carrier was contacted (hit and/or held) by an opponent without reference to whether the ball-carrier went to ground" (Quarrie \& Hopkins, 2008). A HIA tackle was defined as "when a player received a direct/in-direct head impact in the tackle and was subsequently removed from play for a HIA and did not return to play for the remainder of the game" (Tierney et al., 2018b). Only HIA scenarios involving tacklers as the injured player were used for the current study.

Tackles were categorised as either upper body tackles which are defined by the tackler's intended primary contact being above the ball carrier's hip or lower body tackles defined by intended primary contact being at or below the ball carrier's hip (Tierney, Lawler, et al., 2016; Tierney \& Simms, 2017a, 2017b). Tackles were also categorised as either front-on or side-on tackles. Tackles initiated outside the ball carriers estimated peripheral vision (roughly \pm 60 degrees from the direction the ball carrier's head is pointing) were considered side-on tackles (Burger et al., 2016; Garraway et al., 1999). Sideon tackles included tackles from behind (Burger et al., 2016). For side-on tackles, awareness was identified by the ball carrier adjusting their head direction such that the tackler was roughly within the abovementioned \pm 60 degrees during the pre-contact phase of the tackle (see below).

Full details on the case-control cohort are available in Tierney et al. (Tierney et al., 2018b). In brief, videos of HIA cases were obtained from elite level competitions/test series including the Pro 12 (2014-2017), European Rugby Champions Cup (2014-2017), RBS 6 Nations (2014-2017), Guinness Autumn Test Series (2013-2016), the 2015 Rugby World Cup and the 2017 British and Irish Lions Tour. A total of 74 HIA cases were identified (19 upper body and 19 lower body for front-on tackles and 23 upper body and 13 lower body for side-on tackles). A direct head impact was identified in every video even though a HIA can result from an impact to the body (McCrory et al., 2005).

The non-HIA cases consist of tackles from three randomly selected European Rugby Champions Cup games. As a result of this approach, a total of 92 upper body tackles and 30 lower body tackles for front-on tackles and 75 upper body tackles and 36 lower body tackles for side-on tackles were analysed as control cases. Ethical permission was not required as all the data was freely available online similar to other rugby union video analysis studies on head impacts/injury (Montgomery et al., 2016; Tierney, Lawler, et al., 2016).

\subsection{Technical proficiency criteria}

The ball carrier technique characteristics are based on the work of Burger et al. (2016) (Burger et al., 2016). These technical based characteristics were created for front- and side-on tackles based on studies that analysed tackling proficiency in collision sports (Gabbett, 2008; Gabbett \& Kelly, 2007; Gabbett \& Ryan, 2009; Hendricks, Matthews, Roode, \& Lambert, 2014). The criteria were further developed by a specialist group of rugby union coaches, medical personnel and sport scientists.

Each video was analysed by two reviewers together (a Senior Sports Physiotherapist and a Biomechanist). Any differences between reviewers were resolved by a review and discussion until a consensus was reached. Video analysis was conducted by utilising Sports Code (Version 8) which allowed frame-by-frame viewing of the tackle. Reviewers watched the clips as many times as necessary and a minimum of two camera views (25 fps) were available for every tackle. The technical proficiency characteristics were assigned to the three main phases of the tackle (Hendricks et al., 2014); precontact ( $0.5 \mathrm{~s}$ preceding contact), contact (first instance of contact) and post-contact. If a player exhibited a characteristic, they would score 1 for that particular characteristic, otherwise they would score 0 .

\subsection{Statistical analysis}

Statistical analysis was conducted using SPSS (IBM SPSS Statistics for Windows, Version 22.0. Armonk, NY: IBM Corp.). For each ball carrier proficiency characteristic, Pearson's ChiSquare and Cramer's V were calculated (Altman, 1990). Statistical significance was set at $p<0.05$. Statistically significant tackle technique characteristics are regarded as having a higher propensity to result in a HIA for the tackler than that anticipated by chance. A Cramer's V value less than 0.1 , between 0.1 and less than 0.3 , between 0.3 and less than 0.5 and 0.5 or greater were considered indicative of trivial, small, moderate and large Effect Sizes (ES) respectively (Cohen, 1988). 
Table 1. Ball carrier Upper Body Tackle front-on proficiency results for HIA and non-HIA tackles (includes \% occurrence, $\mathrm{p}$ values, Cramer's V and interpretations).

\begin{tabular}{|c|c|c|c|c|c|}
\hline & $\begin{array}{c}\text { HIA } \\
(n=19)\end{array}$ & $\begin{array}{l}\text { Non-HIA } \\
(\mathrm{n}=92)\end{array}$ & $p$ value & Cramer's V & Interpretation \\
\hline \multicolumn{6}{|l|}{ Pre-contact } \\
\hline Eyes Focused on tackler & $13(68 \%)$ & $76(83 \%)$ & 0.16 & 0.13 & Small \\
\hline Shifting the ball away from contact & $5(26 \%)$ & $43(47 \%)$ & 0.10 & 0.16 & Small \\
\hline Body position - Upright to low & $6(31 \%)$ & $44(48 \%)$ & 0.20 & 0.12 & Small \\
\hline Body Position-Straight back & $15(79 \%)$ & $73(79 \%)$ & 0.97 & $<0.01$ & Trivial \\
\hline Head up and forward, eyes open & $14(74 \%)$ & $59(64 \%)$ & 0.42 & 0.08 & Trivial \\
\hline Shuffle or evasive manoeuvre & $3(16 \%)$ & $19(21 \%)$ & 0.63 & 0.05 & Trivial \\
\hline \multicolumn{6}{|l|}{ Contact } \\
\hline Fending into contact & $9(47 \%)$ & $14(15 \%)$ & $<0.01$ & 0.30 & Moderate \\
\hline Side-on into contact & $4(21 \%)$ & $12(13 \%)$ & 0.37 & 0.09 & Trivial \\
\hline Explosiveness on contact & $11(58 \%)$ & $29(31 \%)$ & 0.03 & 0.21 & Small \\
\hline Body position- from low body position up into contact & $3(16 \%)$ & $18(20 \%)$ & 0.70 & 0.04 & Trivial \\
\hline Ball protection & $17(90 \%)$ & $90(98 \%)$ & 0.08 & 0.17 & Small \\
\hline \multicolumn{6}{|l|}{ Post-contact } \\
\hline Leg drive on contact & $11(58 \%)$ & $48(52 \%)$ & 0.65 & 0.03 & Trivial \\
\hline Arm and shoulder usage & $9(47 \%)$ & $40(44 \%)$ & 0.76 & 0.03 & Trivial \\
\hline Go to ground and present ball/offload & $17(90 \%)$ & $89(97 \%)$ & 0.16 & 0.13 & Small \\
\hline
\end{tabular}

Table 2. Ball carrier Upper Body Tackle side-on proficiency results for HIA and non-HIA tackles (includes \% occurrence, $\mathrm{p}$ values, Cramer's V and interpretations).

\begin{tabular}{|c|c|c|c|c|c|}
\hline & $\begin{array}{c}\text { HIA } \\
(n=23)\end{array}$ & $\begin{array}{l}\text { Non-HIA } \\
(n=75)\end{array}$ & $p$ value & Cramer's V & Interpretation \\
\hline \multicolumn{6}{|l|}{ Pre-contact } \\
\hline Aware of tackler (attunement) & $15(65 \%)$ & $45(60 \%)$ & 0.65 & 0.05 & Trivial \\
\hline Shifting the ball away from contact & $13(56 \%)$ & $35(47 \%)$ & 0.41 & 0.08 & Trivial \\
\hline Body position - Upright to low & $5(22 \%)$ & $13(17 \%)$ & 0.63 & 0.05 & Small \\
\hline Body Position-Straight back & $21(91 \%)$ & $66(88 \%)$ & 0.66 & 0.04 & Trivial \\
\hline Head up and forward, eyes open & $15(65 \%)$ & $61(81 \%)$ & 0.11 & 0.16 & Small \\
\hline Shuffle or evasive manoeuvre & $2(9 \%)$ & $17(23 \%)$ & 0.14 & 0.15 & Small \\
\hline \multicolumn{6}{|l|}{ Contact } \\
\hline Fending away from contact & $2(9 \%)$ & $14(19 \%)$ & 0.26 & 0.11 & Small \\
\hline Explosiveness away from contact & $9(39 \%)$ & $25(33 \%)$ & 0.61 & 0.05 & Trivial \\
\hline Ball protection & $22(96 \%)$ & $67(89 \%)$ & 0.36 & 0.09 & Trivial \\
\hline \multicolumn{6}{|l|}{ Post-contact } \\
\hline Leg drive on contact & $8(35 \%)$ & $31(41 \%)$ & 0.57 & 0.06 & Trivial \\
\hline Go to ground and present ball/offload & $21(91 \%)$ & $66(88 \%)$ & 0.66 & 0.04 & Trivial \\
\hline
\end{tabular}

Table 3. Ball carrier Lower Body Tackle front-on proficiency results for HIA and non-HIA tackles (includes \% occurrence, $\mathrm{p}$ values, Cramer's $\mathrm{V}$ and interpretations).

\begin{tabular}{|c|c|c|c|c|c|}
\hline & $\begin{array}{c}\text { HIA } \\
(n=19)\end{array}$ & $\begin{array}{l}\text { Non-HIA } \\
(n=30)\end{array}$ & $\mathrm{p}$ value & Cramer's V & Interpretation \\
\hline \multicolumn{6}{|l|}{ Pre-contact } \\
\hline Eyes Focused on tackler & $18(95 \%)$ & $27(90 \%)$ & 0.56 & 0.08 & Trivial \\
\hline Shifting the ball away from contact & $10(53 \%)$ & $18(60 \%)$ & 0.61 & 0.07 & Trivial \\
\hline Body position - Upright to low & $12(63 \%)$ & $13(43 \%)$ & 0.18 & 0.19 & Small \\
\hline Body Position-Straight back & $15(79 \%)$ & $28(93 \%)$ & 0.13 & 0.21 & Small \\
\hline Head up and forward, eyes open & $17(90 \%)$ & $27(90 \%)$ & 0.95 & $<0.01$ & Trivial \\
\hline Shuffle or evasive manoeuvre & $7(37 \%)$ & $10(33 \%)$ & 0.80 & 0.04 & Trivial \\
\hline \multicolumn{6}{|l|}{ Contact } \\
\hline Fending into contact & $4(21 \%)$ & $3(10 \%)$ & 0.28 & 0.15 & Small \\
\hline Side-on into contact & $5(26 \%)$ & $8(27 \%)$ & 0.98 & $<0.01$ & Trivial \\
\hline Explosiveness on contact & $10(53 \%)$ & $7(23 \%)$ & 0.04 & 0.30 & Moderate \\
\hline Body position- from low body position up into contact & $2(11 \%)$ & $4(13 \%)$ & 0.77 & 0.04 & Trivial \\
\hline Ball protection & $18(95 \%)$ & $29(97 \%)$ & 0.74 & 0.05 & Trivial \\
\hline \multicolumn{6}{|l|}{ Post-contact } \\
\hline Leg drive on contact & $7(37 \%)$ & $11(37 \%)$ & 0.99 & $<0.01$ & Trivial \\
\hline Arm and shoulder usage & $5(26 \%)$ & $4(13 \%)$ & 0.25 & 0.16 & Small \\
\hline Go to ground and present ball/offload & $18(95 \%)$ & $28(93 \%)$ & 0.84 & 0.03 & Trivial \\
\hline
\end{tabular}

\subsection{Reliability}

Thirty tackles (Fifteen front-on and fifteen side-on tackles, including HIA and non-HIA cases) were selected randomly using a number generator (http://www.random.org/). For intra-rater reliability, the two reviewers reanalysed these 30 cases, for each ball carrier proficiency characteristic, at least one week after conducting the initial analysis. For inter-rater reliability, an external reviewer (ex-player) analysed the same 30 cases using the same protocol as the two main reviewers. Intra-rater and inter-rater reliability were assessed using Cohen's Kappa (K). A Cohen's Kappa value higher than 0.8 indicates almost perfect agreement (Landis \& Koch, 1977). For front-on tackles, a Cohen's 
Table 4. Ball carrier Lower Body Tackle side-on proficiency results for HIA and non-HIA tackles (includes \% occurrence, $\mathrm{p}$ values, Cramer's V and interpretations).

\begin{tabular}{|c|c|c|c|c|c|}
\hline & $\begin{array}{c}\text { HIA } \\
(n=13)\end{array}$ & $\begin{array}{l}\text { Non-HIA } \\
(n=36)\end{array}$ & $p$ value & Cramer's V & Interpretation \\
\hline \multicolumn{6}{|l|}{ Pre-contact } \\
\hline Aware of tackler (attunement) & $11(85 \%)$ & $29(81 \%)$ & 0.75 & 0.05 & Trivial \\
\hline Shifting the ball away from contact & $8(62 \%)$ & $25(69 \%)$ & 0.60 & 0.07 & Trivial \\
\hline Body position - Upright to low & $2(15 \%)$ & $4(11 \%)$ & 0.69 & 0.06 & Trivial \\
\hline Body Position-Straight back & $12(92 \%)$ & $35(97 \%)$ & 0.44 & 0.11 & Small \\
\hline Head up and forward, eyes open & $13(100 \%)$ & $33(92 \%)$ & 0.28 & 0.16 & Small \\
\hline Shuffle or evasive manoeuvre & $4(31 \%)$ & $20(56 \%)$ & 0.13 & 0.22 & Small \\
\hline \multicolumn{6}{|l|}{ Contact } \\
\hline Fending away from contact & $1(8 \%)$ & $10(28 \%)$ & 0.14 & 0.21 & Small \\
\hline Explosiveness away from contact & $7(54 \%)$ & $13(36 \%)$ & 0.27 & 0.16 & Small \\
\hline Ball protection & $13(100 \%)$ & $33(92 \%)$ & 0.28 & 0.16 & Small \\
\hline \multicolumn{6}{|l|}{ Post-contact } \\
\hline Leg drive on contact & $4(31 \%)$ & $14(39 \%)$ & 0.60 & 0.07 & Trivial \\
\hline Go to ground and present ball/offload & $13(100 \%)$ & $32(89 \%)$ & 0.21 & 0.18 & Small \\
\hline
\end{tabular}

Kappa value of 0.93 and 0.81 were calculated for intra- and inter-rater reliability, respectively. For side-on tackles, a Cohen's Kappa value of 0.95 and 0.86 were calculated for intra-rater reliability and inter-rater reliability, respectively.

\section{Results}

For front-on upper body tackles (Table 1) only the contact phase influenced HIA causation. The ball carrier characteristics "Explosiveness on contact" ( $p=0.03 ; E S=$ Small) and "fending into contact" ( $p<0.01$, ES = Moderate) had a higher propensity to result in a HIA for the tackler. Fending into contact was exhibited in almost half of all upper body tackle front-on HIA cases (47\%). Additional analysis found that in $67 \%$ of these cases it was the fending arm (upper arm, elbow and forearm), and not the palm of the hand, that contacted the tackler's head. Furthermore, the ball carrier was not legally leading with the palm of the hand in these cases. None of these cases resulted in a foul being given.

No characteristics for side-on upper body tackles (Table 2) had a higher propensity to result in a HIA for the tackler. In $35 \%(n=8)$ of side-on upper body tackles, it was another tackler from the same team that impacted the tackler's head as both team mates collided while attempting to tackle the same ball carrier. In one case, both tacklers received HIAs.

For front-on lower body tackles (Table 3), only "explosiveness on contact" ( $p=0.04$; ES = Moderate) had a higher propensity to result in a HIA for the tackler. No characteristics for side-on lower body tackles (Table 4) had a higher propensity to result in a HIA for the tackler. In one side-on lower body Tackle, it was another tackler from the same team that impacted the other tackler's head.

\section{Discussion}

\subsection{General}

This study utilised match video evidence to identify ball carrier characteristics that have a higher propensity to result in a HIA for the tackler. The results from this study provide an evidence-base at an elite level to assist with the development of strategies to prevent head impacts which result in a HIA. Only two ball carrier proficiency variables that contribute to a high HIA risk for the tackler were identified. In particular, no side-on ball carrier characteristics were identified as having a higher propensity to result in a HIA for the tackler. Tierney et al. (Tierney et al., 2018b) found a number of tackler characteristics that were associated with a high tackler HIA risk. This highlights the importance of effective and safe tackler proficiency characteristics and that focus should be placed on tackler characteristics for prevention strategies, particularly for side-on tackles.

Previous studies have shown that fending has a positive effect on ball carrier tackle outcomes (Hendricks et al., 2014; Tierney et al., 2018c; Wheeler \& Sayers, 2009). One study found that a moderate fend increased the chance of offloading (Hendricks et al., 2014) whilst another study reported that a strong fend compromised the quality of the tackler's positioning (Wheeler \& Sayers, 2009). However, the same study (Wheeler \& Sayers, 2009) also found that the type of fend (e.g. moderate, strong etc) influenced outcomes such as tackle breaks and offloads. In addition to these studies, fending has also been shown to reduce the risk of ball carrier injury (Burger et al., 2016).

Fending into contact was exhibited in almost half of all upper body tackle front-on HIA cases (47\%) and in $67 \%$ of these cases it was not legally executed. According to Law 7 of rugby union, the ball carrier is only permitted to fend off an opponent by using the palm of the hand (Rugby, 2017). As of November 2016, World Rugby added a reckless tackle sanction to the laws of the game by stating that "A player is deemed to have made reckless contact during a tackle or attempted tackle or during other phases of the game if in making contact, the player knew or should have known that there was a risk of making contact with the head of an opponent, but did so anyway. This sanction applies even if the tackle starts below the line of the shoulders. This type of contact also applies to grabbing and rolling/twisting around the head/neck area even if the contact starts below the line of the shoulders (Rugby, 2016)." The minimum and maximum sanction for a reckless tackle is a yellow and red card, respectively. The results of this study agree with this addition to the laws and illustrates the importance of its enforcement. With regards to HIA prevention, coaches should place focus on safe fending during tackle based training drills and referees should be alert to arm-to-head contact during the fend.

"Explosiveness on contact" was a ball carrier characteristic identified as having a higher propensity to result in a HIA for 
the tackler for both upper and lower body front-on tackles. This is consistent with a previous study that identified energy transfer in the tackle as a risk factor for HIAs (Tucker et al., 2017). It is difficult to mitigate against this risk as explosivity is a desirable trait amongst players.

\subsection{Limitations}

The tackle is a dynamic and open phase of play and this must be appreciated when analysing tackling characteristics (Burger et al., 2016; Garraway et al., 1999). The HIA definition utilised in this study was based on a player being removed from the game for a HIA and subsequently not returning to the field of play. This can be considered a strong indication of concussion, but without access to player medical notes, cannot be used as a concussion diagnosis. Access to player medical notes would have clarified this. The control cases only utilised three games and only one team was analysed, meaning the results could be team specific. The non-HIA cases were from elite level club competition match play (European Champions Cup) whereas the HIA dataset includes both elite level club and international match play. Ideally both elite level club and international match play would be included in the control dataset. The analysis was conducted on elite level rugby union games. Although there are no HIAs in amateur and youth level rugby union, the findings may be applicable to this level of play to prevent significant direct head impacts (Tierney et al., 2016). However, further research on tackle characteristics in amateur and youth level rugby union is needed to clarify this. Further work could also model the combination and interaction of other technical characteristics and match situation characteristics, such as tackle speed (Gabbett \& Kelly, 2007; Tucker et al., 2017) and tackle height (Tierney \& Simms, 2018a, 2018b) to allow for an even greater understanding of HIA risk. In order to fully understand the mechanism of head injury, further work should investigate the biomechanics of head impacts (Tierney, Joodaki, et al., 2016; Tierney, Joodaki, et al., 2018; Tierney, Richter, et al., 2018).

\section{Conclusion}

Fending into contact is a ball carrier characteristic than can potentially be dangerous for the tackler. This characteristic was exhibited in $47 \%$ of all upper body Tackle front-on HIA cases. In $67 \%$ of these cases, it was the fending arm (upper arm, elbow and forearm), and not the palm of the hand, that contacted the tacklers head. The results from this study provide an evidence-base at an elite level to assist with the development of HIA prevention strategies. In particular, coaches should place focus on safe fending during tackle based training drills and referees should be alert to arm-to-head contact during the fend. "Explosiveness on contact" was a ball carrier characteristic identified as having a higher propensity to result in a HIA for the tackler for both upper and lower body front-on tackles, though it is difficult to mitigate against this risk. Much fewer ball carrier proficiency variables that result in a HIA for the tackler were identified in comparison to tackler proficiency characteristics that were identified in an earlier study. In particular, no side-on ball carrier characteristics were identified as having a higher propensity to result in a HIA for the tackler. This highlights the importance of effective and safe tackler proficiency characteristics and that focus should be placed more on tackler characteristics for HIA prevention strategies.

\section{Acknowledgements}

The authors would like to thank the Irish Research Council for funding this study and John Buckley for helping with data collection

\section{Disclosure statement}

No potential conflict of interest was reported by the authors.

\section{Funding}

This work was supported by the Irish Research Council;

\section{ORCID}

Gregory J Tierney (D) http://orcid.org/0000-0002-4666-4473

\section{References}

Altman, D. G. (1990). Practical statistics for medical research (pp. 229-277). Boca Raton, FL: CRC press.

Andersen, T. E., Arnason, A., Engebretsen, L., \& Bahr, R. (2004). Mechanisms of head injuries in elite football. British Journal of Sports Medicine, 38(6), 690-696.

Burger, N., Lambert, M. I., Viljoen, W., Brown, J. C., Readhead, C., \& Hendricks, S. (2016). Tackle technique and tackle-related injuries in high-level South African Rugby Union under-18 players: Real-match video analysis. British Journal of Sports Medicine, 50(15), 932-938.

Cohen, J. (1988). Statistical power and analysis for the behavioral sciences (2 ed ed., pp. 7-82). Hillsdale, N.J.: Lawrence Erlbaum Associates, Inc.

Deutsch, M., Kearney, G., \& Rehrer, N. (2007). Time-Motion analysis of professional rugby union players during match-play. Journal of Sports Sciences, 25(4), 461-472.

Fuller, C. W., Brooks, J. H., Cancea, R. J., Hall, J., \& Kemp, S. P. (2007). Contact events in rugby union and their propensity to cause injury. British Journal of Sports Medicine, 41(12), 862-867.

Fuller, C. W., Fuller, G. W., Kemp, S. P. T., \& Raftery, M. (2017). Evaluation of World Rugby's concussion management process: Results from Rugby World Cup 2015. British Journal of Sports Medicine, 51(1), 64-69.

Fuller, C. W., Laborde, F., Leather, R. J., \& Molloy, M. G. (2008). International Rugby Board Rugby World Cup 2007 injury surveillance study. British Journal of Sports Medicine, 42(6), 452-459.

Fuller, C. W., Taylor, A., Kemp, S. P. T., \& Raftery, M. (2017). Rugby World Cup 2015: World Rugby injury surveillance study. British Journal of Sports Medicine, 51(1), 51-57.

Fuller, C. W., Taylor, A., \& Raftery, M. (2015). Epidemiology of concussion in men's elite Rugby-7s (Sevens World Series) and Rugby-15s (Rugby World Cup, Junior World Championship and Rugby Trophy, Pacific Nations Cup and English Premiership). British Journal of Sports Medicine, 49(7), 478-483.

Fuller, G. W., Kemp, S. P., \& Decq, P. (2015). The International Rugby Board (IRB) Pitch Side Concussion Assessment trial: A pilot test accuracy study. British Journal of Sports Medicine, 49(8), 529-535.

Fuller, G. W., Kemp, S. P. T., \& Raftery, M. (2017). The accuracy and reproducibility of video assessment in the pitch-side management of concussion in elite rugby. Journal of Science and Medicine in Sport, 20(3), 246-249. 
Gabbett, T., \& Kelly, J. (2007). Does fast defensive line speed influence tackling proficiency in collision sport athletes?, International Journal of Sports Science \& Coaching, 2(4), 467-472.

Gabbett, T., \& Ryan, P. (2009). Tackling technique, injury risk, and playing performance in high-performance collision sport athletes. International Journal of Sports Science \& Coaching, 4(4), 521-533.

Gabbett, T. J. (2008). Influence of fatigue on tackling technique in rugby league players. The Journal of Strength \& Conditioning Research, 22(2), 625-632.

Gardner, A. J., Iverson, G. L., Quinn, T. N., Makdissi, M., Levi, C. R., Shultz, S. R., ... Stanwell, P. (2015). A preliminary video analysis of concussion in the National Rugby League. Brain Injury, 1-4. doi:10.3109/ 02699052.2015 .1034179

Garraway, W., Lee, A., Macleod, D., Telfer, J., Deary, I. J., \& Murray, G. D. (1999). Factors influencing tackle injuries in rugby union football. British Journal of Sports Medicine, 33(1), 37-41.

Hendricks, S., \& Lambert, M. (2010). Tackling in rugby: Coaching strategies for effective technique and injury prevention. International Journal of Sports Science and Coaching, 5(1), 117-136.

Hendricks, S., \& Lambert, M. (2014). Theoretical model describing the relationship between the number of tackles in which a player engages, tackle injury risk and tackle performance. Journal of Sports Science and Medicine, 13(3), 715.

Hendricks, S., Matthews, B., Roode, B., \& Lambert, M. (2014). Tackler characteristics associated with tackle performance in rugby union. European Journal of Sport Science, 14(8), 753-762.

Hendricks, S., O'Connor, S., Lambert, M., Brown, J., Burger, N., Mc Fie, S., ... Viljoen, W. (2015). Contact technique and concussions in the South African under-18 Coca-Cola Craven Week Rugby tournament. European Journal of Sport Science, 15(6), 557-564.

Hendricks, S., O'Connor, S., Lambert, M., Brown, J. C., Burger, N., Mc Fie, S., ... Viljoen, W. (2016). Video analysis of concussion injury mechanism in under-18 rugby. BMJ Open Sport \& Exercise Medicine, 2(1), e000053.

Hutchison, M. G., Comper, P., Meeuwisse, W. H., \& Echemendia, R. J. (2013). A systematic video analysis of National Hockey League (NHL) concussions, part I: Who, when, where and what?,British Journal of Sports Medicine, 49(8), 547-551.

Landis, J. R., \& Koch, G. G. (1977). The measurement of observer agreement for categorical data. Biometrics, 31(1), 159-174.

McCrory, P., Johnston, K., Meeuwisse, W., Aubry, M., Cantu, R., Dvorak, J., .. Schamasch, P. (2005). Summary and agreement statement of the 2nd International Conference on Concussion in Sport, Prague 2004. British Journal of Sports Medicine, 39(4), 196-204.

Montgomery, C., Blackburn, J., Withers, D., Tierney, G., Moran, C., \& Simms, C. (2016). Mechanisms of ACL injury in professional rugby union: $A$ systematic video analysis of 36 cases. British Journal of Sports Medicine, 1-8. doi:10.1136/bjsports-2016-096425

Quarrie, K. L., \& Hopkins, W. G. (2008). Tackle Injuries in Professional Rugby Union. The American Journal of Sports Medicine, 36(9), 1705-1716.

Rugby, W. (2017). Laws of the game: Law 7. Retrieved from http://laws. worldrugby.org/?law $=7$ \&language $=\mathrm{EN}$

Rugby, W. (2016, Novemebr 2016). Reckless and accidental tackles. Retrieved from http://laws.worldrugby.org/?domain=9\&language=EN

Shuttleworth-Edwards, A. B., Noakes, T. D., Radloff, S. E., Whitefield, V. J., Clark, S. B., Roberts, C. O., ... Case, S. E. (2008). The comparative incidence of reported concussions presenting for follow-up management in South African Rugby Union. Clinical Journal of Sport Medicine, 18(5), 403-409.

Tierney, G. J., Denvir, K., Farrell, G., \& Simms, C. K. (2018a). Does player time-in-game affect tackle technique in elite level rugby union?, Journal of Science and Medicine in Sport, 21(2), 221-225.

Tierney, G. J., Denvir, K., Farrell, G., \& Simms, C. K. (2018b). The effect of tackler technique on head injury assessment risk in elite rugby union. Medicine \& Science in Sports \& Exercise, 50(3), 603-608.

Tierney, G. J., Denvir, K., Farrell, G., \& Simms, C. K. (2018c). The effect of technique on tackle gainline success outcomes in elite level rugby union. International Journal of Sports Science \& Coaching, 13(1), 16-25.

Tierney, G. J., Joodaki, H., Krosshaug, T., Forman, J. L., Crandall, J. R., \& Simms, C. K. (2016). The kinematics of head impacts in contact sport: An initial assessment of the potential of model based image matching. ISBSConference Proceedings Archive. pp. 108-111 10.1016/j. puhe.2015.10.038

Tierney, G. J., Joodaki, H., Krosshaug, T., Forman, J. L., Crandall, J. R., \& Simms, C. K. (2018). Assessment of model-based image-matching for future reconstruction of unhelmeted sport head impact kinematics. Sports Biomechanics, 17(1), 33-47.

Tierney, G. J., Krosshaug, T., Wilson, F., \& Simms, C. K. (2015). An Assessment of a Novel Approach for Determining the Player Kinematics in Elite Rugby Union Players. Paper presented at the IRCOBI Conference, Lyon, France. pp. 180-181. 10.1002/net.21589

Tierney, G. J., Lawler, J., Denvir, K., McQuilkin, K., \& Simms, C. (2016). Risks associated with significant head impact events in elite rugby union. Brain Injury, 30(11), 1350-1361.

Tierney, G. J., Richter, C., Denvir, K., \& Simms, C. K. (2018). Could lowering the tackle height in rugby union reduce ball carrier inertial head kinematics?, Journal of Biomechanics. Advance Online Publication. doi:10.1016/j.jbiomech.2018.02.023

Tierney, G. J., \& Simms, C. K. (2017a). The effect of intended primary contact location on tackler head impact risk. Paper presented at the IRCOBI Conference, Antwerp, Belgium. pp. 703-704.

Tierney, G. J., \& Simms, C. K. (2017b). The effects of tackle height on inertial loading of the head and neck in Rugby Union: A multibody model analysis. Brain Injury, 31(13-14), 1925-1931.

Tierney, G. J., \& Simms, C. K. (2018a). Can tackle height influence tackle gainline success outcomes in elite level rugby union?,International Journal of Sports Science \& Coaching. Advance Online Publication. doi:10.1177/1747954117751345

Tierney, G. J., \& Simms, C. K. (2018b). Can tackle height influence head injury assessment risk in elite rugby union?,Journal of Science and Medicine in Sport. Advance Online Publication. doi:10.1016/j. jsams.2018.05.010

Tucker, R., Raftery, M., Kemp, S., Brown, J., Fuller, G., Hester, B., ... Quarrie, K. (2017). Risk factors for head injury events in professional rugby union: $A$ video analysis of 464 head injury events to inform proposed injury prevention strategies. British Journal of Sports Medicine, 51(15), 1152-1157.

Union, R. F. England Professional Rugby Injury Surveillance Project 2018. Retrieved from: http://www.englandrugby.com/mm/Document/General/ General/01/32/91/95/InjurySurveillanceReport2016-17_English.pdf.

Wheeler, K., \& Sayers, M. (2009). Contact skills predicting tackle-breaks in rugby union. International Journal of Sports Science \& Coaching, 4(4), 535-544. 